\title{
USE OF THE HUNT FILTER TO OPTIMIZE THE DETERMINA- TION OF IMPULSE-RESPONSE FUNCTIONS OF INDIVIDUAL COMPONENT PARTS OF FLOW-INJECTION MANIFOLDS
}

\author{
I.C. VAN NUGTEREN-OSINGA, M. BOS and W.E. VAN DER LINDEN* \\ University of Twente, Laboratory for Chemical Analysis-CT, P.O. Box 217, NL-7500 AE \\ Enschede (The Netherlands)
}

(Received 8th December 1988)

\section{SUMMARY}

The dispersion behaviour of the various individual parts making up a flow-injection manifold can be expressed by means of impulse-response functions. These functions can be determined by deconvolution of the response curves obtained with and without the part concerned. Special attention is paid to a procedure to decrease the influence of noise. It is shown that good results can be obtained with a Hunt filter which operates in the Fourier domain.

In a previous paper [1], it was demonstrated how an impulse-response function of a single module in a flow-injection system can be determined. The proposed procedure consists of a deconvolution of response curves obtained with and without the relevant module. This deconvolution step is performed in the Fourier or frequency domain, in which it is a simple division. It was shown, however, that the result of this division is seriously affected by the fact that all measurements are corrupted with noise. The influence is mostly noticeable in the higher frequency range where the absolute values of the amplitudes are relatively small. Since on back-transformation these higher frequencies are less important for the general shape of the impulse-response function, an appropriate filter must be chosen. In general, the choice of such a filter is a matter of trial and error and a small number of filters are tested. The Hunt filter [2] appears to be the most successful. Originally, the Hunt filter was used for image restoration; the simplified one-dimensional version appears to be suitable for the determination of the impulse-response functions of individual components of the flow-injection manifold. 


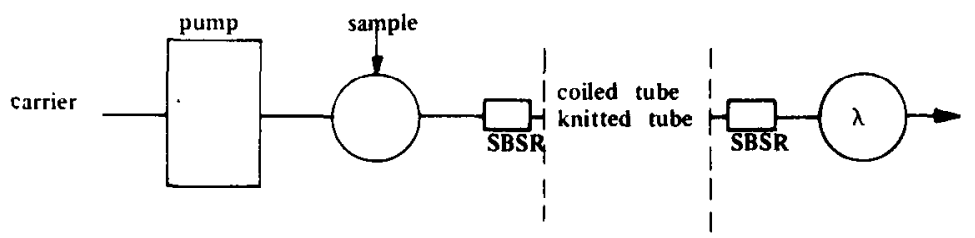

Fig. 1. Manifold for measuring the dispersion in tubes.

\section{EXPERIMENTAL}

\section{Chemicals}

All chemicals were of analytical-reagent grade. A dye stock solution was prepared by dissolving $0.400 \mathrm{~g}$ of bromothymol blue in $25 \mathrm{ml}$ of ethanol and diluting to $100 \mathrm{ml}$ with $0.01 \mathrm{M}$ sodium borate. Sample solutions were prepared by 100 -fold dilution of this stock solution with $0.01 \mathrm{M}$ sodium borate. The carrier solution was $2 \times 10^{-5} \mathrm{M}$ sodium borate.

\section{Apparatus}

The manifold used is depicted schematically in Fig. 1. The peristaltic pump was a Gilson Minipuls II. Polyethylene tubing was used. The injection valve was of a rotary type (Rheodyne) and controlled by the computer. The injection volume was about $100 \mu \mathrm{l}$. Tubes ( $30 \mathrm{~mm} \times 1.2 \mathrm{~mm}$ i.d.) packed with glass beads (diameter $0.65-0.9 \mathrm{~mm}$ ) were used to accomplish enhanced radial mixing wherever required, i.e., at the entrance of each element. The detector was a Zeiss PMQ III variable-wavelength photometer equipped with an $80-\mu \mathrm{l}$ cell (Helma).

An Apple Ile computer was used for controlling the experiments and for collecting the data. The curves were sampled at a rate of $5 \mathrm{~s}^{-1}$. The fast Fourier transformation uses 256 points, so the frequency resolution is $0.0195 \mathrm{~Hz}$. The modules tested were $1 \mathrm{~m}$ of tubing $(0.78 \mathrm{~mm}$ i.d.) coiled with a coil diameter of $5 \mathrm{~mm}$ and a $1 \mathrm{~m}$ tube $(0.89 \mathrm{~mm}$ i.d.) that was 'knitted' [3]. The response curves were measured at a constant pumping rate of $1.8 \mathrm{ml} \mathrm{min}^{-1}$.

\section{RESULTS AND DISCUSSION}

For the determination of an impulse-response function of a certain module, first the response of the flow-injection system $f(t)$, without this module is measured and subsequently the response with the module, $g(t)$, is measured. The mathematical procedure can be depicted as follows:

$$
f(t) \rightarrow F F T \rightarrow F(f)
$$

$$
\rightarrow G(f) / F(f)=H(f) \rightarrow \text { filter } \rightarrow \text { inverse } F F T \rightarrow h(t)
$$

$g(t) \rightarrow F F T \rightarrow G(f)$ 
FFT denotes the use of fast Fourier transform algorithm [4], and $F(f)$ and $G(f)$ are the transformed curves, respectively. In Fig. 2 the responses are shown for the system with and without the module.

Because $H(f)$ is obtained by division, noise on $F(f)$ and $G(f)$ has a great effect on the result, particularly when the values of $F(f)$ and $G(f)$ are small, as can be seen at the higher frequencies. Therefore, an appropriate method of filtering is essential.

Various filters have been investigated. The simplest filter tested is rectangular, where only the first 16 unchanged frequencies are used in the backtransformation, but this leads to unacceptable artefacts in the form of sidelobes. As these artefacts are due to the abrupt truncation, some kind of weighting was introduced. Here, the first five frequencies, which mainly determine the shape of the function in the time domain, are not changed but the contribution from each of the following eleven frequencies is gradually decreased by multiplying both the real part and the imaginary part by a logarithmically decreasing factor. Although this filter gave acceptable results, the shape of the filter is rather arbitrary. The use of a Hunt filter may eliminate this subjective element. The filter action can be depicted by the following mathematical procedure:

$H(f)=G(f) F^{*}(f) /\left[F(f) F^{*}(f)+k\right]$

where $F^{*}(f)$ is the complex conjugate of $F(f)$ and $k$ is a constant, the value of which is related to the noise. The filtering action of the Hunt filter is minimal for those frequencies where the curves have large amplitudes and which are only slightly affected by noise, whereas its action is maximal at those frequencies where the amplitudes are small and the result obtained on division is mainly determined by noise.

The remaining question is how to select an appropriate value of $k$. To solve this problem, the following procedure is adopted. First, a baseline is measured
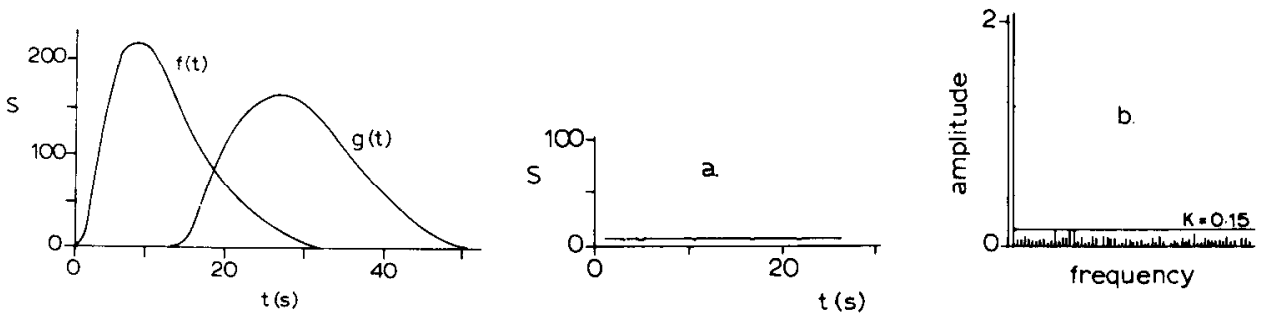

Fig. 2. Response curves of the flow-injection system without extra coil inserted, $f(t)$, and with coil (coil diameter $5 \mathrm{~mm}$ ) inserted, $g(t)$.

Fig. 3. (a) Baseline and (b) the Bode plot of discrete Fourier transform of the baseline; a value of $k$ is also depicted. 
and the result is transformed to the frequency domain. The value of the amplitudes gives an indication of the noise and hence of a suitable value of $k$, which should be slightly larger then the noise level (Fig. 3). It is also possible to estimate the value of $k$ from the Bode plot of the transformed signal of the system equipped with, for example, the coil with a diameter of $5 \mathrm{~mm}$. Therefore, it should be realized that the high frequencies represent mainly the noise and so the values of the amplitudes of these frequencies can also be used to obtain a first estimate of the value of $k$. In the Bode plot of the transformed signal of the system of the coil with a coil diameter of $5 \mathrm{~mm}$ (Fig. 4), a value of $k$ is depicted.

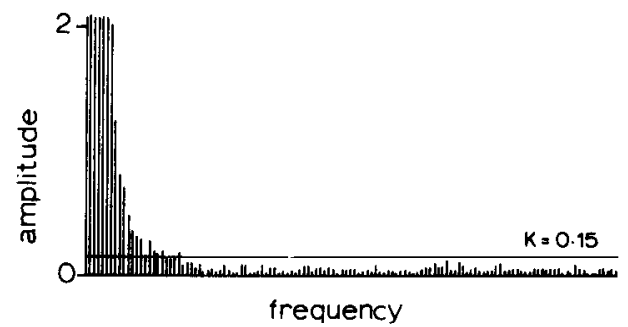

Fig. 4. A detail of the Bode plot of the response of the system with the coil (coil diameter $5 \mathrm{~mm}$ ); a value of $k$ is also depicted.
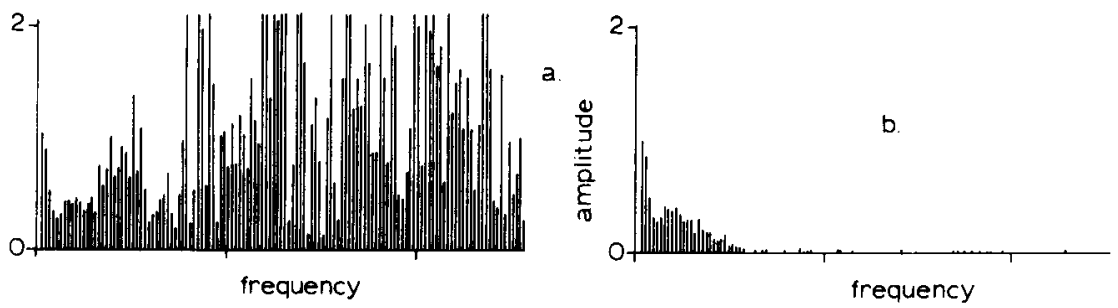

Fig. 5. (a) Bode plot of discrete Fourier transform of the impulse-response function obtained by a division of the Fourier transforms of the output function, $g(t)$, and the input function, $f(t)$, without using the Hunt filter. (b) Bode plot of discrete transform of the impulse-response function of the coil; the Hunt filter is used $(k=0.20)$.

TABLE 1

Improvement of the impulse-response functions determined with different values of $k$

\begin{tabular}{llll}
\hline$k=$ new & $k=$ old & $\begin{array}{l}5 \text {-mm } \\
\text { coil }\end{array}$ & $\begin{array}{l}\text { Knitted } \\
\text { coil }\end{array}$ \\
\hline 0.15 & 0.10 & 5.06 & 5.52 \\
0.20 & 0.15 & 1.95 & 2.13 \\
0.25 & 0.20 & 0.98 & 1.06 \\
0.30 & 0.25 & 0.58 & 0.61 \\
0.35 & 0.30 & 0.38 & 0.39 \\
\hline
\end{tabular}




\section{TABLE 2}

Calculated functions which roughly fit the measured responses of the system

\begin{tabular}{lllll}
\hline Function & $a$ & $n_{1}$ & $n_{2}$ & $\tau$ \\
\hline $\begin{array}{l}\text { Function that fits the response of the } \\
\text { system without a module: } f(t)\end{array}$ & 0.5 & 4 & 4 & 1.45 \\
$\begin{array}{l}\text { Function that fits the response of the } \\
\text { system with the 5-mm coil: } g(t)\end{array}$ & 0.05 & 3 & 4 & 1.70 \\
$\begin{array}{l}\text { Function that fits the response of the } \\
\text { system with the knitted coil: } g(t)\end{array}$ & 0.1 & 4 & 6 & 1.50 \\
\hline
\end{tabular}

\section{TABLE 3}

Relationship between the amplitude of the noise and the value of $k$

\begin{tabular}{|c|c|c|c|c|}
\hline \multirow[t]{2}{*}{ Parameter } & \multicolumn{2}{|l|}{ Value of $k$} & \multicolumn{2}{|c|}{ Improvement } \\
\hline & 5-mm coil & Knitted coil & 5-mm coil & Knitted coil \\
\hline \multicolumn{4}{|l|}{ Maximal amplitude of the noise: 1} & \\
\hline $\begin{array}{cl}\text { Improvement } & k_{0.25}, k_{0.20} \\
& k_{0.30}, k_{0.35} \\
& k_{0.34}, k_{0.40}\end{array}$ & & & $\begin{array}{l}1.75 \\
1.13 \\
0.79\end{array}$ & $\begin{array}{l}0.64 \\
0.34 \\
0.20\end{array}$ \\
\hline Chosen value of $k$ & 0.35 & 0.25 & & \\
\hline $\begin{array}{l}\text { Maximal amplitude of the noise: } 2 \\
\text { First indication of the value of } k\end{array}$ & 0.25 & 0.25 & & \\
\hline $\begin{array}{ll}\text { Improvement: } & k_{0.40} k_{0.45} \\
& k_{0.45}, k_{0.50}\end{array}$ & & & $\begin{array}{l}1.15 \\
0.62\end{array}$ & $\begin{array}{l}0.72 \\
0.25\end{array}$ \\
\hline Chosen value of $k$ & 0.45 & 0.40 & & \\
\hline $\begin{array}{l}\text { Maximal amplitude of the noise: } 3 \\
\text { First indication of the value of } k\end{array}$ & 0.40 & 0.40 & & \\
\hline $\begin{array}{ll}\text { Improvement: } & k_{0.50}, k_{0.55} \\
& k_{0.55}, k_{0.60}\end{array}$ & & & $\begin{array}{l}1.32 \\
0.62\end{array}$ & $\begin{array}{l}1.00 \\
0.38\end{array}$ \\
\hline Chosen value of $k$ & 0.55 & 0.55 & & \\
\hline
\end{tabular}

The effect of the use of the Hunt filter on the noise is illustrated in Fig. 5 . After inverse transformation the result is judged, when the influence of the noise is still found to be high, a larger value of $k$ can be chosen. This procedure can be repeated by a stepwise increase in $k$ in increments of 0.05 and comparing the result of the new impulse-response function $\left(h_{k=\text { new }}\right)$ with the old function ( $h_{k=\text { old }}$ ) according to the following criterion:

improvement $=\sum\left[h_{k=\text { old }}(t)-h_{k=\text { new }}(t)\right]^{2}$

When the improvement is $<1.0$, no difference between the old and new impulse-response functions can be discerned. The improvement in the impulseresponse functions of two coils for several $k$ values is summarized in Table 1 . To determine the relationship between the value of $k$ and the noise, white noise 
is superimposed on calculated curves. The curves fit roughly the measured responses of the system without and with the different modules. The curves are calculated with the following function:

$$
\begin{aligned}
f(t) \text { or } g(t)= & {[a / \tau]\left[(t / p \tau)^{n_{1}-1} /\left(n_{1}-1\right) !\right] \exp (t / \tau) } \\
& +[(1-a) / \tau]\left[(t / \tau)^{n_{2}-1} /\left(n_{2}-1\right) !\right] \exp (t / \tau)
\end{aligned}
$$

The values of the constants of the functions used are summarized in Table 2. The maximal amplitudes of the noise were 1, 2 and 3 (for comparison, the maximal amplitude of the calculated function that fits with the response of the system without module is 224 ). As before, the first indication of the value of $k$ is found in the Bode plots of the transformed functions. And again, when the
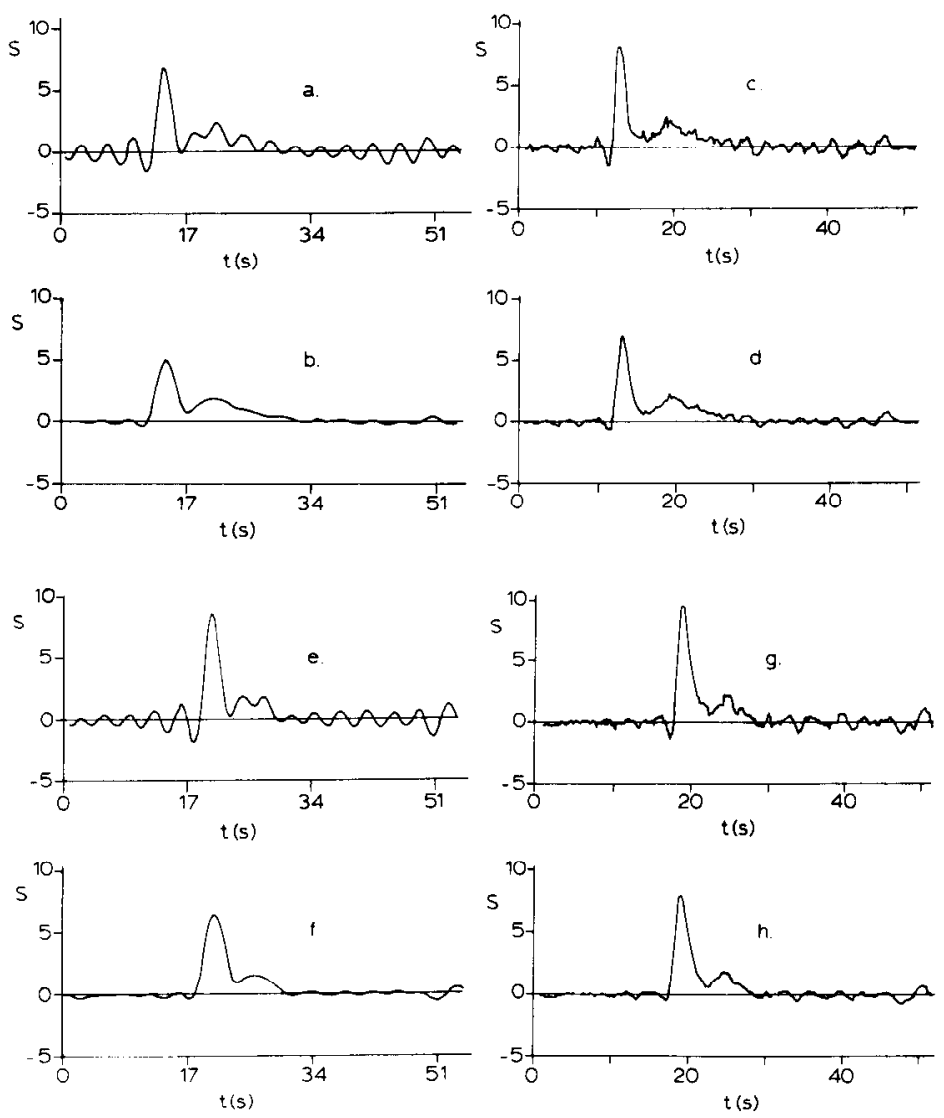

Fig. 6. Impulse-response curves for coiled tube, length $1 \mathrm{~m}$, tube i.d. $0.78 \mathrm{~mm}$ and coil diameter 5 $\mathrm{mm}$, flow-rate $1.8 \mathrm{ml} \mathrm{min}^{-1}$ : (a) rectangular filter; (b) weighting filter; (c) Hunt filter, $k=0.10$; (d) Hunt filter, $k=0.20$. Impulse-response curves for knitted tube, length $1 \mathrm{~m}$, tube i.d. $0.86 \mathrm{~mm}$ and flow-rate $1.8 \mathrm{ml} \mathrm{min}^{-1}$ : (e) rectangular filter; (f) weighting filter; (g) Hunt filter, $k=0.10$; (h) Hunt filter, $k=0.25$. 
improvement is $<1.0$, the impulse-response function is found. As can be seen in Table 3, the value of $k$ is related to the maximal amplitude of the noise.

The results for the different filters are shown in Fig. 6. It is obvious that the use of a rectangular filter leads to the appearance of spurious side-lobes, whereas a weighting filter flattens and broadens the impulse-response function. With the use of the Hunt filter this feature is much smaller.

\section{Conclusions}

The Hunt filter deals with the noise in the determination of the impulseresponse functions of individual components of flow-injection manifolds in an elegant manner. The filter action is adapted to the noise characteristics, in contrast to the filter action of the weighting and rectangular filters. The Hunt filter also uses some information about the high frequencies, whereas the rectangular and the weighting filters ignore this information completely. The Hunt filter also causes much less flattening and broadening of the impulse-response function than a weighting filter.

The authors thank Professor dr. A. Bagchi and Professor ir. E.W. Gröneveld for helpful discussions on some theoretical aspects, and Dr. ir. B. Vandeginste for suggesting the use of the Hunt filter. These investigations were supported (in part) by the Netherlands Foundation of Chemical Research (SON) with financial aid from the Netherlands' Technology Foundation.

\section{REFERENCES}

1 I.C. van Nugteren-Osinga, M.Bos and W.E. van der Linden, Anal. Chim. Acta, 214 (1988) 77.

2 B.R. Hunt, IEEE Trans. Comput., 22 (1973) 805.

3 U. Neue and H. Engelhardt, Chromatographia, 15 (1982) 403.

4 W.H. Press, B.P. Flannery, S.A. Teukolsky and W.T. Vetterling, Numerical Recipes, Cambridge University Press, Cambridge, 1986. 